JOURNAL OF SYNCHROTRON RADIATION

ISSN 1600-5775

Received 30 August 2021

Accepted 25 November 2021

Edited by K. Kvashnina, ESRF - The European Synchrotron, France

Keywords: molten salt method; actinide immobilization; $\mathrm{Nd}_{2} \mathrm{Zr}_{2} \mathrm{O}_{7} ;$ X-ray absorption spectroscopy.

Supporting information: this article has supporting information at journals.iucr.org/s
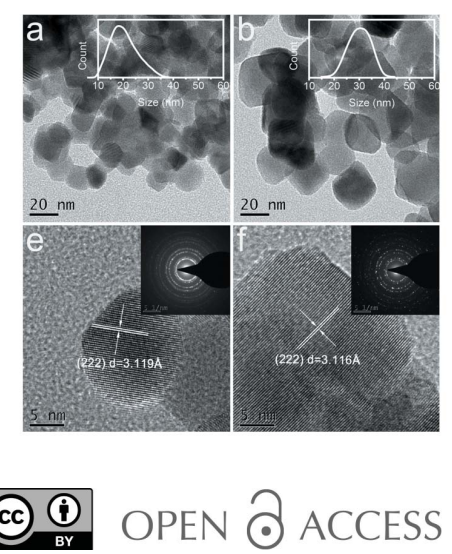

\section{Controllable sites and high-capacity immobilization of uranium in $\mathrm{Nd}_{2} \mathrm{Zr}_{2} \mathrm{O}_{7}$ pyrochlore}

\author{
Jian Sun, ${ }^{\mathrm{a}, \mathrm{b}}$ Jing Zhou, ${ }^{\mathrm{a}}$ Zhiwei Hu, ${ }^{\mathrm{c}}$ Ting-Shan Chan, ${ }^{\mathrm{d}}$ Renduo Liu, ${ }^{\mathrm{a}}$ Haisheng $\mathrm{Yu}^{\mathrm{a}}$ \\ Linjuan Zhang ${ }^{a, b *}$ and Jian-Qiang Wang ${ }^{a, b *}$

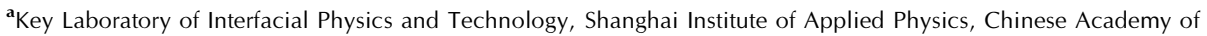 \\ Sciences, Shanghai 201800, People's Republic of China, ' $U$ University of Chinese Academy of Sciences, Beijing 100049, \\ People's Republic of China, 'Max Planck Institute for Chemical Physics of Solids, Nöthnitzer Strasse 40, 01187 Dresden, \\ Germany, and ${ }^{\mathbf{d}}$ National Synchrotron Radiation Research Center, 101 Hsin-Ann Road, Hsinchu 30076, Taiwan. \\ *Correspondence e-mail: zhanglinjuan@sinap.ac.cn, wangjianqiang@sinap.ac.cn
}

As potential nuclear waste host matrices, two series of uranium-doped $\mathrm{Nd}_{2} \mathrm{Zr}_{2} \mathrm{O}_{7}$ nanoparticles were successfully synthesized using an optimized molten salt method in an air atmosphere. Our combined X-ray diffraction, Raman and X-ray absorption fine-structure (XAFS) spectroscopy studies reveal that uranium ions can precisely substitute the $\mathrm{Nd}$ site to form an $\mathrm{Nd}_{2-x} \mathrm{U}_{x} \mathrm{Zr}_{2} \mathrm{O}_{7+\delta}(0 \leq x \leq 0.2)$ system and the $\mathrm{Zr}$ site to form an $\mathrm{Nd}_{2} \mathrm{Zr}_{2-y} \mathrm{U}_{y} \mathrm{O}_{7+\delta}$ $(0 \leq y \leq 0.4)$ system without any impurity phase. With increasing $\mathrm{U}$ concentration, there is a phase transition from pyrochlore $(F d \overline{3} m)$ to defect fluorite $(F m \overline{3} m)$ structures in both series of U-doped $\mathrm{Nd}_{2} \mathrm{Zr}_{2} \mathrm{O}_{7}$. The XAFS analysis indicates that uranium exists in the form of high-valent $\mathrm{U}^{6+}$ in all samples. To balance the extra charge for substituting $\mathrm{Nd}^{3+}$ or $\mathrm{Zr}^{4+}$ by $\mathrm{U}^{6+}$, additional oxygen is introduced accompanied by a large structural distortion; however, the $\mathrm{Nd}_{2} \mathrm{Zr}_{1.6} \mathrm{U}_{0.4} \mathrm{O}_{7+\delta}$ sample with high $\mathrm{U}$ loading $(20 \mathrm{~mol} \%)$ still maintains a regular fluorite structure, indicating the good solubility of the $\mathrm{Nd}_{2} \mathrm{Zr}_{2} \mathrm{O}_{7}$ host for uranium. This study is, to the best of our knowledge, the first systematic study on $\mathrm{U}$-incorporated $\mathrm{Nd}_{2} \mathrm{Zr}_{2} \mathrm{O}_{7}$ synthesized via the molten salt method and provides convincing evidence for the feasibility of accurately immobilizing $\mathrm{U}$ at specific sites.

\section{Introduction}

The disposal of actinide-rich nuclear waste is a key issue for the development of sustainable nuclear energy (Ewing, 2011; Abdou et al., 2018; Finkeldei et al., 2020; Liu et al., 2021). The search for high-capacity, low-cost and radiation-tolerant materials that can be used as host materials for nuclear wastes has been a major research interest for a long time. Pyrochlore oxides have received considerable attention in immobilizing actinides owing to their desirable physical-chemical properties, including their high thermal stability, high chemical durability, and strong resistance to radiation damage (Abdou et al., 2018; Fuentes et al., 2018; Finkeldei et al., 2020). Pyrochlore, ideally $A_{2} B_{2} \mathrm{O}_{7}$, can accommodate a variety of chemical compositions, in which typically a trivalent cation occupies the $A$ sites coordinated by eight oxygen atoms and tetravalent cation occupies the $B$ sites coordinated by six oxygen atoms (Marlton et al., 2021; Talanov \& Talanov, 2021). Among them, zirconate pyrochlore is the most important class of host materials due to its remarkable resistance to amorphization under ion beam irradiation ( $\mathrm{Lu}, \mathrm{Shu}$, Chen et al., 2018; Lu, Shu, Shao et al., 2018; Lu et al., 2019; Shu et al., 2017). Previous studies have demonstrated that actinides can be effectively incorporated into the zirconate pyrochlore matrix 
at both the $A$ and $B$ sites (Blackburn et al., 2021; Jafar et al., 2015; Lu et al., 2017; Shu et al., 2016; Wang et al., 2021), but many impurities, such as $\mathrm{ZrO}_{2}, \mathrm{U}_{3} \mathrm{O}_{8}, \mathrm{ThO}_{2}$ and other complex uranium oxides, were introduced at high actinide concentrations, and the stability of the pyrochlore was reduced. Moreover, the immobilization capacity, local structure and valence state of the incorporated actinides (i.e. their speciation) have not been fully explored in previous reports. Realizing the rational manipulation of the phase structure and substituting sites in zirconate pyrochlore, as well as developing low-cost and sustainable synthetic methods, remains a tremendous challenge, which has practical significance in the application of immobilizing actinides.

The conventional method for the preparation of zirconate pyrochlore is a solid-state reaction, which normally results in inhomogeneity and impurities in the final phases (Shu et al., 2016). To improve the chemical homogeneity and phase accuracy of the system, several wet-chemistry routes have been employed for the preparation of these zirconium-based pyrochlore materials, which involves the mixing of reactant precursors and fast mass transport at the molecular level, resulting in better compositional homogeneity and controlled stoichiometric ratio of the obtained samples. However, subsequent sintering is generally carried out at temperatures above $1400^{\circ} \mathrm{C}$ for more than $48 \mathrm{~h}$, which is of high cost and not suitable for large-scale waste disposal ( $\mathrm{Lu}, \mathrm{Shu}$, Chen et al., 2018). Principally, molten salt synthesis is a potential methodology that can lower the reaction temperature due to faster mass transport. In previous work (Mao et al., 2009; Gupta \& Mao, 2021), $\mathrm{La}_{2} \mathrm{Zr}_{2} \mathrm{O}_{7}$ pyrochlore ceramic was synthesized via the molten salt method by heating mixtures at $650^{\circ} \mathrm{C}$ for $6 \mathrm{~h}$. However, until now, only limited work has been reported on actinide-incorporated pyrochlore via the molten salt method (Abdou et al., 2018, 2019; Wang et al., 2020). Neodymium zirconate has also been proposed as a potential ceramic matrix for the immobilization of nuclear waste; therefore, in this work, we chose $\mathrm{Nd}_{2} \mathrm{Zr}_{2} \mathrm{O}_{7}$ as a suitable host material for studying uranium immobilization via a molten salt process. We successfully synthesized two series of $\mathrm{Nd}_{2} \mathrm{Zr}_{2} \mathrm{O}_{7}$ (NZO) nanoparticles doped with different $\mathrm{U}$ concentrations by an optimized molten salt method and achieved precise control of doping at either $\mathrm{Nd}$ or $\mathrm{Zr}$ sites to form $\mathrm{Nd}_{2-x} \mathrm{U}_{x} \mathrm{Zr}_{2} \mathrm{O}_{7+\delta}$ or $\mathrm{Nd}_{2} \mathrm{Zr}_{2-y} \mathrm{U}_{y} \mathrm{O}_{7+\delta}$, respectively. X-ray diffraction, Raman and transmission electron microscopy were used to characterize the crystalline structure, phase transition and morphology in U-doped NZO nanoparticles. XAFS was used to obtain detailed information on the oxidation state and local environments of metal ions.

\section{Experimental methods and characterization}

\subsection{Reagents}

The reagents used in this experiment include $\mathrm{Nd}\left(\mathrm{NO}_{3}\right)_{3} \cdot 6 \mathrm{H}_{2} \mathrm{O} \quad(99.9 \%), \quad \mathrm{ZrO}\left(\mathrm{NO}_{3}\right)_{2} \cdot 2 \mathrm{H}_{2} \mathrm{O} \quad(99.5 \%)$, $\mathrm{UO}_{2}\left(\mathrm{NO}_{3}\right)_{2} \cdot 6 \mathrm{H}_{2} \mathrm{O}, \mathrm{NH}_{4} \mathrm{OH}(28 \%), \mathrm{NaNO}_{3}(99.9 \%)$ and
$\mathrm{KNO}_{3}$ (99.9\%). All reagents are used as received without further purification.

\subsection{Material synthesis}

All samples were synthesized by an innovative molten salt method (Mao et al., 2009). In the first step, the singlesource composite precursor material was synthesized by the coprecipitation method. The original reactants contained $\mathrm{Nd}\left(\mathrm{NO}_{3}\right)_{3} \cdot 6 \mathrm{H}_{2} \mathrm{O}, \mathrm{ZrO}\left(\mathrm{NO}_{3}\right)_{2} \cdot 2 \mathrm{H}_{2} \mathrm{O}$ and $\mathrm{UO}_{2}\left(\mathrm{NO}_{3}\right)_{2} \cdot 6 \mathrm{H}_{2} \mathrm{O}$. The reactants were measured stoichiometrically as $(5-\alpha) \mathrm{mmol} \mathrm{Nd}\left(\mathrm{NO}_{3}\right)_{3} \cdot 6 \mathrm{H}_{2} \mathrm{O}, 5 \mathrm{mmol} \quad \mathrm{ZrO}\left(\mathrm{NO}_{3}\right)_{2} \cdot 2 \mathrm{H}_{2} \mathrm{O}$ and $\alpha \mathrm{mmol} \mathrm{UO}_{2}\left(\mathrm{NO}_{3}\right)_{2} \cdot 6 \mathrm{H}_{2} \mathrm{O}$ corresponding to $A$ site uranium doping, and $5 \mathrm{mmol} \mathrm{Nd}\left(\mathrm{NO}_{3}\right)_{3} \cdot 6 \mathrm{H}_{2} \mathrm{O},(5-\beta) \mathrm{mmol}$ $\mathrm{ZrO}\left(\mathrm{NO}_{3}\right)_{2} \cdot 2 \mathrm{H}_{2} \mathrm{O}$ and $\beta \mathrm{mmol} \mathrm{UO}_{2}\left(\mathrm{NO}_{3}\right)_{2} \cdot 6 \mathrm{H}_{2} \mathrm{O}$ corresponding to $B$ site uranium doping. All reagents were dissolved in $200 \mathrm{ml}$ deionized water and stirred at $400 \mathrm{rev} \mathrm{min}^{-1}$. Then, $200 \mathrm{ml}$ of dilute ammonia $\left[\mathrm{NH}_{4} \mathrm{OH}: \mathrm{H}_{2} \mathrm{O}\right.$ $=1: 9(v / v)]$ solution was dropped into the solution, forming a flocculent and exhibiting stratification. The stabilized precipitate was centrifuged out and dried in an oven at $80^{\circ} \mathrm{C}$ for $24 \mathrm{~h}$ to obtain a composite homogeneous composite precursor $(2-x) \mathrm{Nd}(\mathrm{OH})_{3} \cdot 2 \mathrm{ZrO}(\mathrm{OH})_{2} \cdot x \mathrm{UO}_{2}(\mathrm{OH})_{2} \cdot n \mathrm{H}_{2} \mathrm{O}$ and $\left.2 \mathrm{Nd}(\mathrm{OH})_{3} \cdot(2-y) \mathrm{ZrO}(\mathrm{OH})_{2} \cdot y \mathrm{UO}_{2}(\mathrm{OH})_{2} \cdot n \mathrm{H}_{2} \mathrm{O}\right]$. In the second step, NZO and uranium-doped NZO were synthesized by the molten salt method. $0.35 \mathrm{~g}$ of the precursor, $2.55 \mathrm{~g}$ of $\mathrm{NaNO}_{3}$ and $3.03 \mathrm{~g}$ of $\mathrm{KNO}_{3}$ were placed into an agate mortar and ground for 30 minutes. The fine mixture was then placed in a corundum crucible and annealed at $750^{\circ} \mathrm{C}$ for $6 \mathrm{~h}$ with a heating rate of $5^{\circ} \mathrm{C} \mathrm{min}^{-1}$. After natural cooling, the mixture was soaked and washed with deionized water five times to remove the residual molten salt. Finally, the sample was dried at $80^{\circ} \mathrm{C}$ for $12 \mathrm{~h}$ to obtain the product. By controlling the stoichiometric ratio of the initial reactants, $\mathrm{NZO}$ and U-substituted NZO materials were obtained, including compounds $\mathrm{NZO}, \quad \mathrm{Nd}_{1.9} \mathrm{U}_{0.1} \mathrm{Zr}_{2} \mathrm{O}_{7+\delta} \quad(A-0.1)$, $\mathrm{Nd}_{1.8} \mathrm{U}_{0.2} \mathrm{Zr}_{2} \mathrm{O}_{7+\delta}(A-0.2), \quad \mathrm{Nd}_{1.6} \mathrm{U}_{0.4} \mathrm{Zr}_{2} \mathrm{O}_{7+\delta}(A-0.4)$, $\mathrm{Nd}_{2} \mathrm{Zr}_{1.9} \mathrm{U}_{0.1} \mathrm{O}_{7+\delta}(B-0.1), \mathrm{Nd}_{2} \mathrm{Zr}_{1.8} \mathrm{U}_{0.2} \mathrm{O}_{7+\delta}(B-0.2)$ and $\mathrm{Nd}_{2} \mathrm{Zr}_{1.6} \mathrm{U}_{0.4} \mathrm{O}_{7+\delta}(B-0.4)$.

\subsection{Characterization}

The crystal structure was analysed by powder X-ray diffraction spectroscopy (XRD) on a Bruker D8 advanced AXS diffractometer with $\mathrm{Cu} K \alpha(\lambda=1.54178 \AA)$ irradiation and micro-Raman spectroscopy on a Horiba LabRAM HR Evolution with laser wavelength of $633 \mathrm{~nm}$. The lattice parameters were obtained by Rietveld refinement using GSAS II software (version 4300) (Toby \& Von Dreele, 2013). The ideal pyrochlore structure model was used, assuming the $8 \mathrm{a}$ and $48 \mathrm{f}$ sites fully occupied. The morphology was characterized by scanning electron microscopy (SEM; Zeiss LEO 1530VP) and transmission electron microscopy (TEM; FEI Tecnai G2 F20 S-TWIN). The X-ray absorption spectra experiments were performed at BL14W1 in the Shanghai Synchrotron Radiation Facility (SSRF) and BL16A1 in the National Synchrotron Radiation Research Center (NSRRC). The storage ring of SSRF was operated at $3.5 \mathrm{GeV}$ with a maximum current of 
$260 \mathrm{~mA}$. Using a Si (111) double-crystal monochromator, the data collection was carried out in transmission mode for the $\mathrm{Zr}$ $K$-edge with an ionization chamber and in fluorescence mode for the $\mathrm{U} L_{3}$-edge with a Lytle detector. The storage ring of NSRRC was operated at $1.5 \mathrm{GeV}$ with a maximum current of $360 \mathrm{~mA}$. Using a double-crystal monochromator, the sample was placed in an argon atmosphere, and the data collection was carried out in fluorescence mode for the $\mathrm{Nd} L_{3}$-edge with a Lytle detector. All data were collected at ambient temperature. The XAFS data were analysed using the program Demeter (Ravel \& Newville, 2005). For extended $\mathrm{X}$-ray absorption fine-structure (EXAFS) data fitting of $\mathrm{Zr}-\mathrm{K}$, the Fourier transform window of the $k$ space was of the sine type in the range 3.0-12.0 $\mathrm{A}$, and the sine type of the $R$ space was in the range 1.0-4.0 $\AA$. The amplitude reduction factor $S_{0}^{2}$ was fixed as 0.75 during EXAFS fitting, based on the fitting result for the known structure of $\mathrm{Zr}$ foil. For EXAFS data fitting of $\mathrm{U}-L_{3}$, the Fourier transform window for $k$ space was of the sine type in the range $2.0-8.5 \AA$ and sine type for $R$ space in the range $1.0-5.0 \AA$. The amplitude reduction factor $S_{0}^{2}$ was set to 0.9 (Zhang et al., 2018).

\section{Results and discussion}

\subsection{X-ray diffraction}

The crystal structures of pure $\mathrm{Nd}_{2} \mathrm{Zr}_{2} \mathrm{O}_{7}(\mathrm{NZO})$ and $\mathrm{U}$ doped NZO materials synthesized by the optimized molten salt method were characterized by powder X-ray diffraction.
As displayed in Fig. 1(a), the diffraction peaks of the asprepared NZO sample are in good agreement with the reflections of a typical pyrochlore structure (space group $F d \overline{3} m$; PDF: 78-1617). However, the superlattice peaks of (111), (311), (331) and (511) for the NZO sample are relatively weak, indicating relatively poor crystallinity and the possible existence of defective fluorite structures (space group: $F m \overline{3} m$ ) (Hagiwara et al., 2019). Rietveld refinement of the XRD pattern demonstrates the purity of the NZO sample with a pyrochlore structure [Fig. 1(b)], and the parameters are summarized in Table S1 of the supporting information.

The U-doped NZO samples have a similar XRD pattern as the pyrochlore structure, supporting the introduction of uranium into $A$ or $B$ sites to generate $\mathrm{Nd}_{2-x} \mathrm{U}_{x} \mathrm{Zr}_{2} \mathrm{O}_{7+\delta}$ or $\mathrm{Nd}_{2} \mathrm{Zr}_{2-y} \mathrm{U}_{y} \mathrm{O}_{7+\delta}$, respectively (Gupta et al., 2016). Compared with that of NZO, the diffraction patterns of all U-doped NZO samples show increasing broadening with increasing uranium substitution level. Such broadening XRD patterns could be ascribed to an increase in lattice distortions induced by the different effective ionic radii of the $\mathrm{U}(0.73 \AA$ for six-fold coordination and $0.86 \AA$ for eight-fold coordination), $\mathrm{Nd}$ (1.11 $\AA$ for eight-fold coordination) and $\mathrm{Zr}(0.72 \AA$ for six-fold coordination) ions (Wang et al., 2021; Ziolkowski, 1985). This may even lead to possible phase transitions in the sample with high $\mathrm{U}$ content. In the $\mathrm{Nd}_{1.6} \mathrm{U}_{0.4} \mathrm{Zr}_{2} \mathrm{O}_{7+\delta}(A-0.4)$ sample, as marked in Fig. 1(a), second-phase $\mathrm{UO}_{3}$ (PDF: 45-0856) was observed, and the diffraction peaks in the high-angle area split, indicating the existence of other oxides, such as $\mathrm{Nd}_{0.5} \mathrm{Zr}_{0.5} \mathrm{O}_{1.75}$ (PDF: 78-1289) or $\mathrm{Nd}_{0.2} \mathrm{Zr}_{0.8} \mathrm{O}_{1.9}$ (PDF:
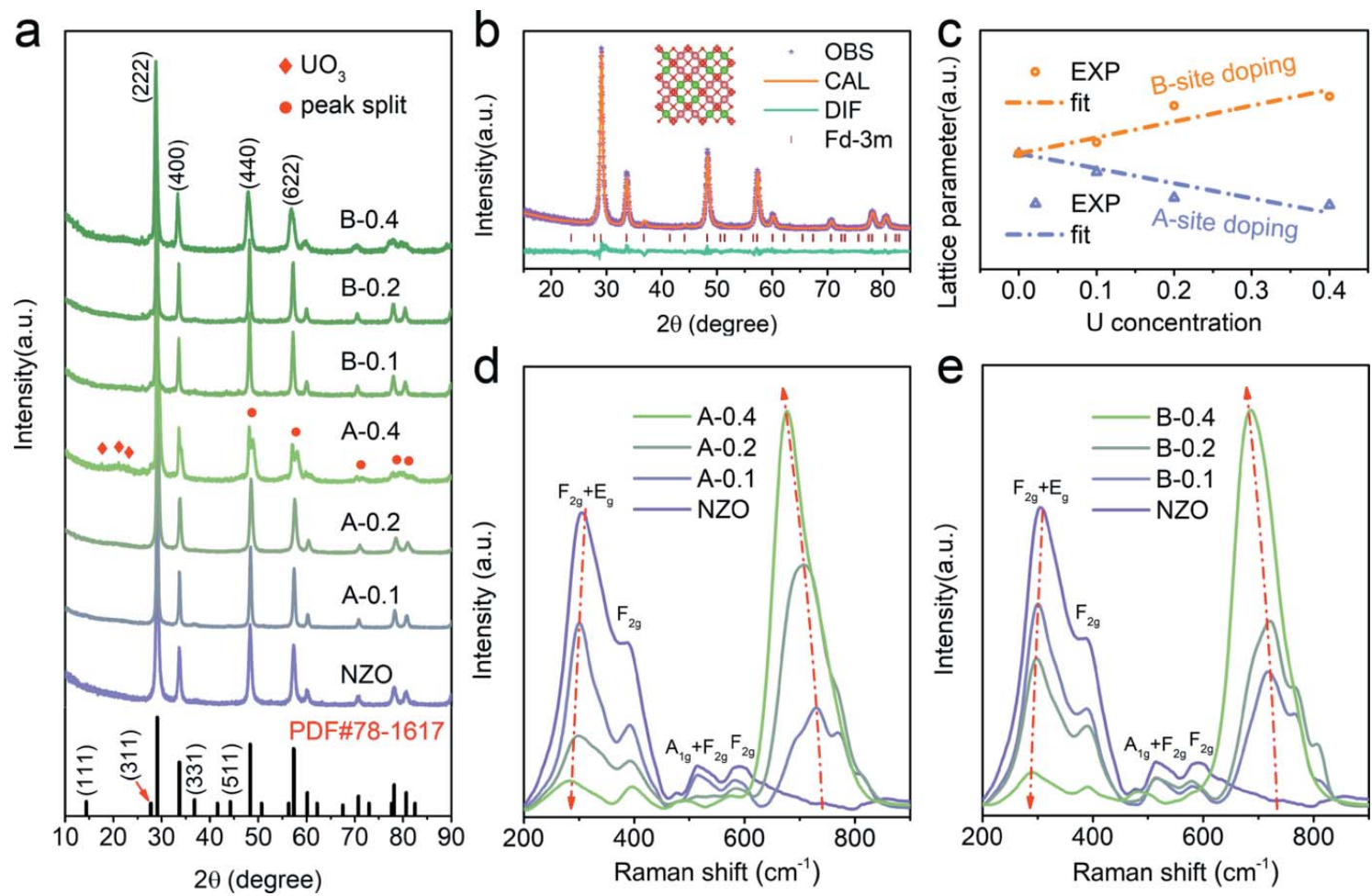

Figure 1

Structural characterization. (a) XRD profiles of $\mathrm{Nd}_{2} \mathrm{Zr}_{2} \mathrm{O}_{7}, \mathrm{Nd}_{2-x} \mathrm{U}_{x} \mathrm{Zr}_{2} \mathrm{O}_{7+\delta}(A-0.1, A-0.2, A-0.4)$ and $\mathrm{Nd}_{2} \mathrm{Zr}_{2-y} \mathrm{U}_{y} \mathrm{O}_{7+\delta}(B-0.1, B-0.2, B-0.4)$. (b) Rietveld refinement of XRD profile for $\mathrm{Nd}_{2} \mathrm{Zr}_{2} \mathrm{O}_{7}$ (NZO). (c) Evolution of the fitted lattice parameters with the U concentrations. Raman spectra of (d) $\mathrm{Nd}_{2-x} \mathrm{U}_{x} \mathrm{Zr}_{2} \mathrm{O}_{7+\delta}$ samples and $(e) \mathrm{Nd}_{2} \mathrm{Zr}_{2-y} \mathrm{U}_{y} \mathrm{O}_{7+\delta}$ samples. 
78-1301). As shown in Fig. 1(c), we clearly observed an opposite trend in the lattice parameters, which shrink in $\mathrm{Nd}_{2-x} \mathrm{U}_{x} \mathrm{Zr}_{2} \mathrm{O}_{7+\delta}$ samples but expand in $\mathrm{Nd}_{2} \mathrm{Zr}_{2-y} \mathrm{U}_{y} \mathrm{O}_{7+\delta}$ samples with increasing uranium content. Considering that the effective radius of $\mathrm{U}^{6+}$ ions is between that value of $\mathrm{Nd}^{3+}$ and $\mathrm{Zr}^{4+}$ ions, the shrinkage or expansion of lattice parameters is easy to understand, which supports that uranium ions can precisely replace either $\mathrm{Nd}$ or $\mathrm{Zr}$ sites in $\mathrm{NZO}$ hosts, as expected. Quantitative structural parameters are summarized in Table $\mathrm{S} 1$, and the $x-\mathrm{O}_{48 f}$ positional parameters in $\mathrm{Nd}_{2-x} \mathrm{U}_{x} \mathrm{Zr}_{2} \mathrm{O}_{7+\delta}$ and $\mathrm{Nd}_{2} \mathrm{Zr}_{2-y} \mathrm{U}_{y} \mathrm{O}_{7+\delta}$ increase regularly with increasing uranium content (except for $A-0.4$ ), implying that the crystal structure undergoes a phase transition from the pyrochlore phase to the defect fluorite phase due to the distortion of the $\mathrm{ZrO}_{6}$ octahedron (Wang et al., 2018). However, $A-0.4$ does not conform to the rule due to the appearance of an impurity phase and phase separation, indicating that the introduction of uranium at the $A$ site by the molten salt method has reached the limit. Clearly, it is much easier for uranium ions to accommodate at $\mathrm{Zr}$ sites than at $\mathrm{Nd}$ sites. For this type of substitution, no significant phase impurities can be observed. The transition from ordered to disordered pyrochlore phase and defect fluorite phase cannot be well distinguished by XRD alone. However, these results can be supplemented by Raman spectroscopy.

\subsection{Raman spectroscopy}

The sensitivity of Raman spectroscopy to oxygen displacements makes it widely used to distinguish pyrochlore and fluorite structures (Mączka et al., 2009; Pokhrel et al., 2016; Sanjuán et al., 2011). In fluorite $(F m \overline{3} m)$, only one Raman active mode is expected. In a perfect pyrochlore $(F d \overline{3} m)$, there are six Raman active vibrational modes: $T=A_{1 g}+E_{g}+4 F_{2 g}$ (Turner et al., 2017), in which $A_{1 g}$ belongs to $\mathrm{O}-B-\mathrm{O}$ bending vibration $\left(\sim 520 \mathrm{~cm}^{-1}\right), E_{g}$ is mainly attributed to the $\mathrm{O}-\mathrm{B}-\mathrm{O}$ bending vibration and $B-\mathrm{O}$ stretching vibration $\left(\sim 300 \mathrm{~cm}^{-1}\right)$, and $F_{2 g}$ is the $\mathrm{O}-B-\mathrm{O}_{6}$ octahedral vibration and $\mathrm{O}-A-\mathrm{O}_{48 f}$ bending vibration $\left(\sim 300 \mathrm{~cm}^{-1}, \quad 400 \mathrm{~cm}^{-1}, \quad 500 \mathrm{~cm}^{-1}\right.$, $600 \mathrm{~cm}^{-1}$ ) (Niu et al., 2019; Park et al., 2018; Zhang et al., 2010). As shown in Figs. 1(d) and 1(e), the Raman spectra of NZO were in good agreement with the peaks of the pyrochlore phase. With increasing $\mathrm{U}$ doping amount, the intensity of the Raman spectra in $\mathrm{Nd}_{2-x} \mathrm{U}_{x} \mathrm{Zr}_{2} \mathrm{O}_{7+\delta}$ and $\mathrm{Nd}_{2} \mathrm{Zr}_{2-y} \mathrm{U}_{y} \mathrm{O}_{7+\delta}$ begins to weaken and shifts to low frequency, which is consistent with Maya Abdou's result (Abdou et al., 2018). These changes reflect the increase in the structural disorder degree, especially for anions (Zhang et al., 2014). In particular, the peak intensity decreases to its lowest at $A-0.4$ and $B-0.4$, suggesting that the pyrochlore phase was gradually transformed into a disordered fluorite phase. In addition, $A-0.1$ and $B-0.1$ exhibit three additional Raman active sites $\left(\sim 720 \mathrm{~cm}^{-1}, 770 \mathrm{~cm}^{-1}, 820 \mathrm{~cm}^{-1}\right)$, which may be related to the distorted $B-\mathrm{O}_{6}$ octahedron induced by the incorporated $\mathrm{U}$ cations (Garg et al., 2008; Tang et al., 2020). Similar modes were found in other uranium compounds (Gregg et al., 2013; Manara \& Renker, 2003; Palacios \& Taylor, 2000; Abdou et al.,
2018; Tang et al., 2020), such as $\mathrm{U}_{3} \mathrm{O}_{8}, \mathrm{UO}_{3}$ and $\mathrm{UO}_{2}{ }^{2+}$ clusters. In $\mathrm{U}_{3} \mathrm{O}_{8}$ and $\mathrm{UO}_{3}, 700 \mathrm{~cm}^{-1}$ is attributed to the stretching of $\mathrm{U}-\mathrm{O}$, and $760 \mathrm{~cm}^{-1}$ is related to the symmetric stretching of $\mathrm{O}=\mathrm{U}=\mathrm{O}$, despite the slightly lower frequency offset. Through semiquantitative analysis of the Raman peak intensity at $700-800 \mathrm{~cm}^{-1}$, we can obtain the corresponding relationship between the doping level of uranium and the structural transformation. The intensity of the peaks at 700$800 \mathrm{~cm}^{-1}$ increases significantly with increasing U substitution, which indicates that uranium ions can be successfully introduced into the parent phase and form a stable conformation. On the other hand, compared with that of $A-0.2$, the three Raman active sites $\left(\sim 720 \mathrm{~cm}^{-1}, 770 \mathrm{~cm}^{-1}, 820 \mathrm{~cm}^{-1}\right)$ are well maintained in the $B-0.2$ sample, implying that the structural incorporation of uranium ions at the $B$ site is better than that at the $A$ site. When the $\mathrm{U}$ concentration reaches $20 \mathrm{~mol} \%$ of the substituted site, both $A-0.4$ and $B-0.4$ show significant structural evolution, which is consistent with the XRD results (Gupta et al., 2016).

\subsection{Morphological characterization}

The morphology of all samples was characterized by SEM and TEM. The elemental distributions (Figure S2) and molar ratios (Table S2) of all samples were detected by SEM-EDS, which indicated good elemental uniformity in the parent material. As shown in Fig. 2(a), the NZO sample has a polyhedral structure with dimensions of $\sim 20 \mathrm{~nm}$. This good uniformity is attributed to the fast mass transfer of the reaction components at the atomic scale, which benefits from the optimized molten salt method (Dargaud, Cormier et al., 2010; Liu et al., 2013).

Compared with the morphology of the NZO parent phase, the characteristics of the U-doped NZO samples show obvious changes with increasing $U$ concentration. The particle sizes of $A-0.1$ and $B-0.1$ are significantly larger than that of NZO. Such differences reflect that the incorporation of uranium affects the initial growth mode, and a higher content of U may further lead to disordered structures and phase transitions, as confirmed by XRD and Raman results. The crystal grains of $A-0.2$ evolve into a spherical shape, while $B-0.2$ still maintains a uniform particle size distribution with a cubic structure. When the uranium content continues to increase, the particles in $A-0.4$ begin to aggregate and grow into irregular masses, while the particles in $B-0.4$ have a good dispersion despite evolving into irregular shapes, supporting that $\mathrm{U}$ doping at $A$ sites is much more different. Importantly, the inset in Fig. 2(d) shows a bimodal size distribution, indicating a kind of phase separation in $A-0.4$, consistent with the XRD result. Well defined lattice fringes were detected by high-resolution transmission electron microscopy (HRTEM), and the lattice fringe spacing corresponding to the (222) planes of $\mathrm{Nd}_{2-x} \mathrm{U}_{x} \mathrm{Zr}_{2} \mathrm{O}_{7+\delta}$ and $\mathrm{Nd}_{2} \mathrm{Zr}_{2-y} \mathrm{U}_{y} \mathrm{O}_{7+\delta}$ have opposite change trends, which is consistent with the XRD results. The inset selected-area electron diffraction (SAED) patterns of all samples show clear polycrystalline diffraction rings, corresponding to the three strong peaks of the pyrochlore or 


\section{actinide physics and chemistry}

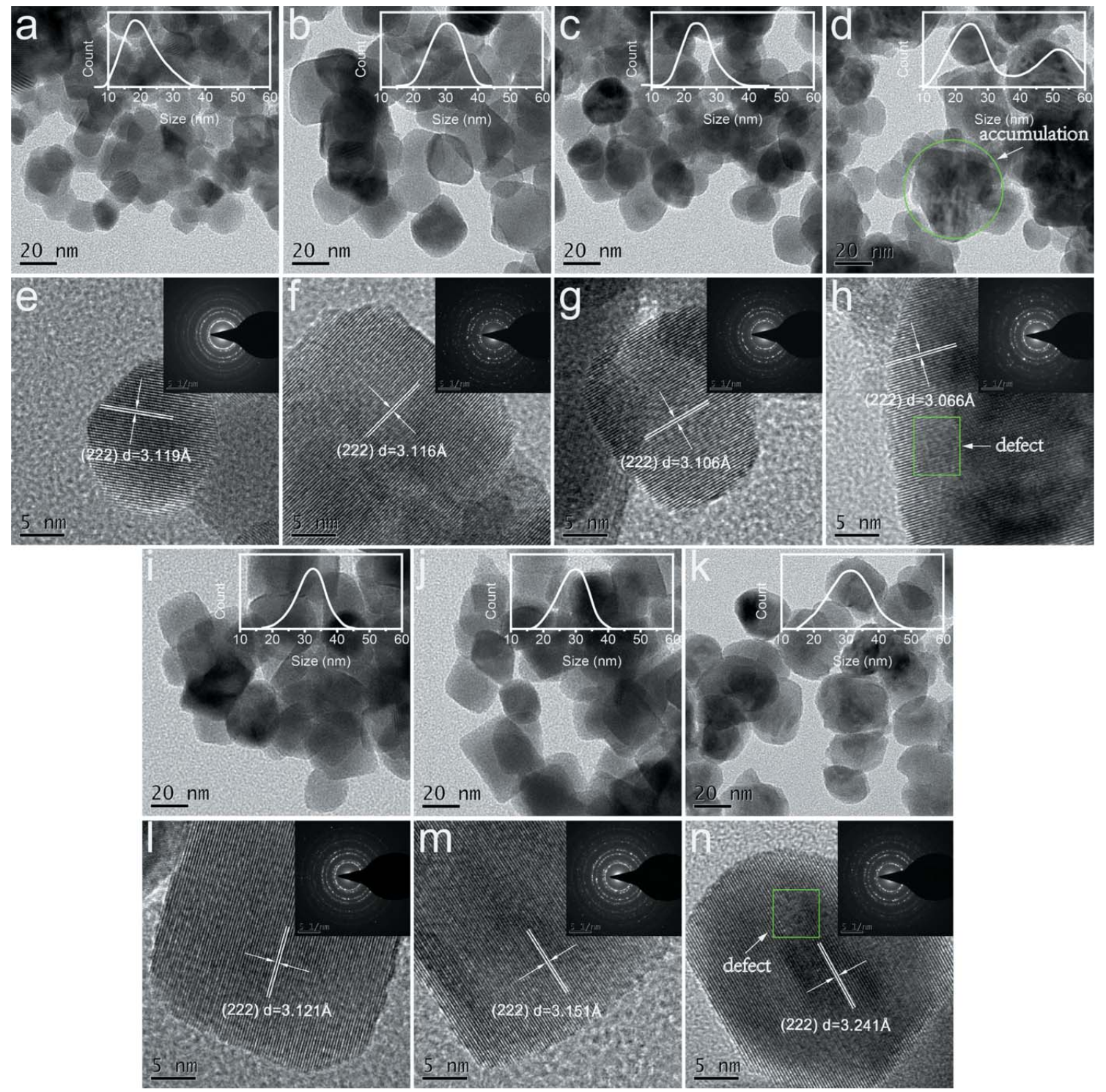

Figure 2

TEM images and HR-TEM results of U-doped NZO samples. Panels $(a, e)$ refer to the NZO samples; $(b, f),(c, g),(d, h)$ refer to the $A-0.1, A-0.2$ and $A-0.4$ samples, respectively; and $(i, l),(j, m)$ and $(k, n)$ refer to the $B-0.1, B-0.2$ and $B-0.4$ samples, respectively; additionally, the illustration shows the corresponding SAED result.

defect fluorite phase. In addition, obvious defects can be observed in the HRTEM for the $A-0.4$ and $B-0.4$ samples [Figs. 2(h), 2(n)].

\subsection{Local electronic and crystal structure}

$\mathrm{X}$-ray absorption fine structure (XAFS) is highly sensitive to the local electronic and crystal structures and therefore has been widely used in actinide compounds (Sun et al., 2019; Zhang et al., 2018; Zhang, Su et al., 2016; Zhang, Zhou et al., 2016; Hu et al., 1998). Fig. 3(a) shows the X-ray absorption near-edge structure (XANES) spectra of the $\mathrm{U} L_{3}$-edge for all U-doped NZO samples, together with spectra of $\mathrm{UO}_{2}$ and $\gamma$ $\mathrm{UO}_{3}$ as a $\mathrm{U}^{4+}$ reference and a $\mathrm{U}^{6+}$ reference, respectively. The energy position of $\mathrm{U} L_{3}$-edge XANES for $\mathrm{U}$-doped NZO samples is basically the same as that of the $\gamma-\mathrm{UO}_{3}$ standard, indicating a $\mathrm{U}^{6+}$ state in all samples. However, to achieve charge balance, the introduction of high-valent $U$ for substituting $\mathrm{Nd}^{3+}$ or $\mathrm{Zr}^{4+}$ sites should induce a large number of cation vacancies or additional oxygen, along with higher structural disorder (Abdou et al., 2018; Finkeldei et al., 2020). It is important to note that the XANES spectral features can be affected by the valence state, local symmetry and structural disorder. Previous works reported that the energy position of spectral features is affected by structural disorder with shifts of approximately $\pm 0.5-1.0 \mathrm{eV}$ (Conradson et al., 2003, 2004). The high structural disorder around high-valent $U$ may explain the wide main peak of $U L_{3}$-edge XANES and the reason that no significant differences were observed between the $\mathrm{Nd}_{2-x} \mathrm{U}_{x} \mathrm{Zr}_{2} \mathrm{O}_{7+\delta}$ and $\mathrm{Nd}_{2} \mathrm{Zr}_{2-y} \mathrm{U}_{y} \mathrm{O}_{7+\delta}$ samples in Fig. 3(a). 

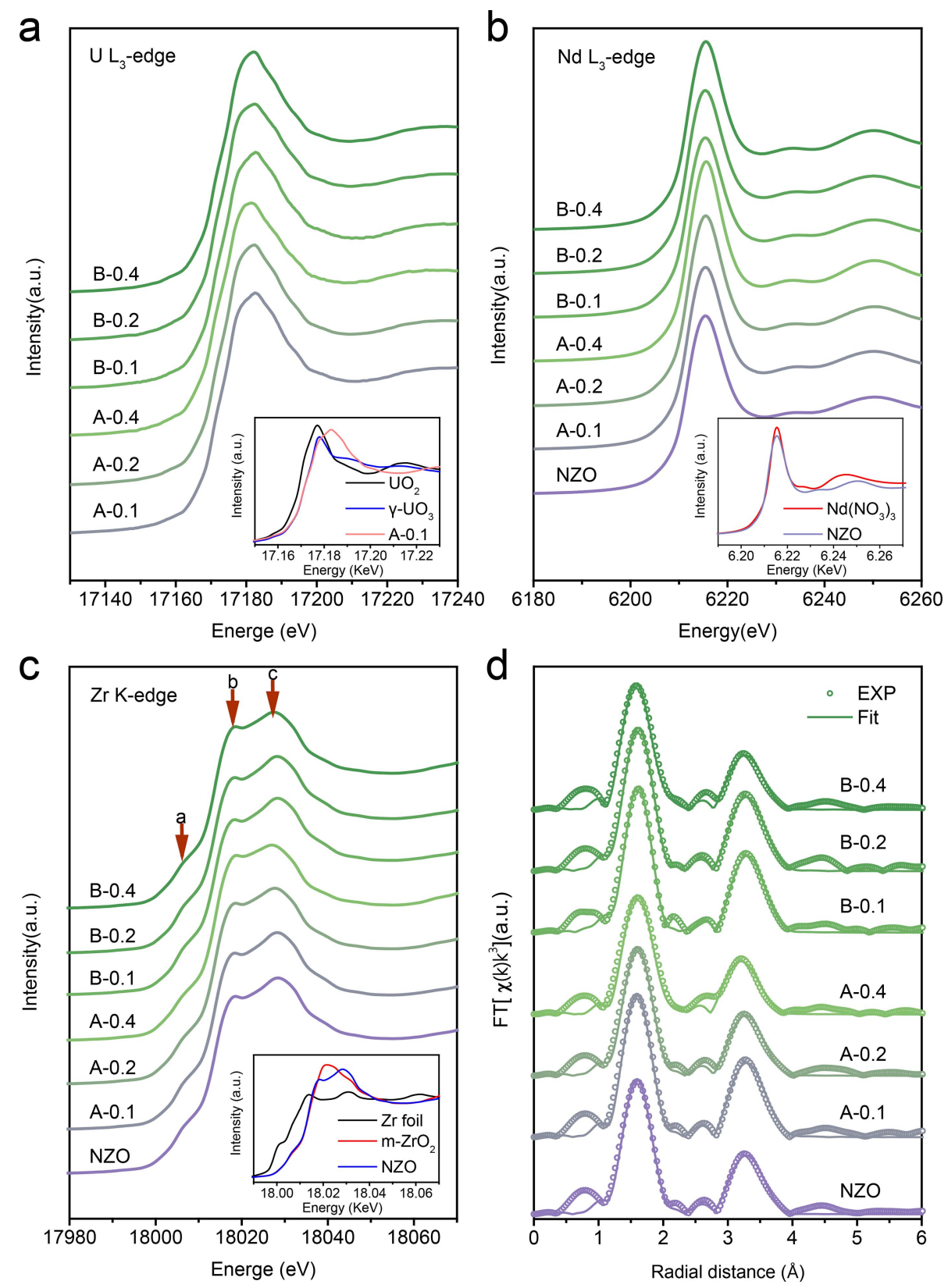

Figure 3

XAFS data of the NZO and U-doped NZO samples. $(a) \mathrm{U}-L_{3},(b) \mathrm{Nd}-L_{3}$ and $(c) \mathrm{Zr} K$-edge XANES spectra of U-doped NZO samples, $(d)$ Fourier transform (FT) of $Z r$ redge EXAFS data and their corresponding fits in $R$ space.

To detect the bonding between uranium and oxygen ligands in U-doped NZO, we performed an extended X-ray absorption fine-structure (EXAFS) analysis of the $\mathrm{U} L_{3}$-edge by using Fourier transform (FT). Figure S3 shows the $k^{3}$-weighted $\chi(k)$ signal in the U-doped NZO sample and its corresponding $\mathrm{U}$ $L_{3}$-edge FT. The broad peak at $\sim 1.5 \AA$ in the $R$ space arising from the $\mathrm{U}-\mathrm{O}$ coordination shell indicates that there is a high oxygen disorder around the $\mathrm{U}$ site. Table $\mathrm{S} 3$ shows that this disorder $\left(\sigma^{2}\right)$ reaches its highest value at $A-0.4$ and $B-0.4$. At the same time, the coordination number decreases as the uranium concentration increases. It is worth noting that, for the U-O path, $\mathrm{Nd}_{2-x} \mathrm{U}_{x} \mathrm{Zr}_{2} \mathrm{O}_{7+\delta}$ has a shorter bond length than $\mathrm{Nd}_{2} \mathrm{Zr}_{2-y} \mathrm{U}_{y} \mathrm{O}_{7+\delta}$. This difference implies that the molten salt method realizes the doping of uranium at the $A$ and $B$ sites of the NZO matrix material and produces a slight difference in the oxygen coordination of uranium.

To assess the structural changes of $\mathrm{Nd}$ and $\mathrm{Zr}$ affected by dopant $\mathrm{U}$ ions in U-doped NZO samples, Nd- $L_{3}$ and $\mathrm{Zr}-K$ XANES measurements were also performed. The $\mathrm{Nd} L_{3}$-edge XANES spectra of all samples exhibit a similar shape, as shown in Fig. 3(b), and the white line energy position is very close to that of the $\mathrm{Nd}\left(\mathrm{NO}_{3}\right)_{3}$ reference, demonstrating the $3+$ valence state of the $\mathrm{Nd}$ ions. In the case of an increase in the valence by one, the white line maximum would shift by $7-8 \mathrm{eV}$ to higher energies (Lissner et al., 1994; Hu et al., 1997). In a typical pyrochlore, $\mathrm{Nd}$ coordinates with eight oxygens to form a hexahedral structure, and it contains two different crystallographic oxygen sites: $\mathrm{O}_{8 b}$ and $\mathrm{O}_{48 f}$. Among them, $\mathrm{O}_{48 f}$ has special properties concerning the adjustment of oxygen disorder and structural distortion in pyrochlore (Hagiwara et al., 2019; Martin et al., 2009). Previous studies pointed out that the introduction of high-valent ions will cause the $8 a$ oxygen vacancies to be partially filled and cause the anion disorder of $\mathrm{Gd}_{2} \mathrm{Zr}_{x} \mathrm{Ti}_{2-x} \mathrm{O}_{7+\delta}$ pyrochlore (Nästren et al., 2009). In the $\mathrm{Nd} L_{3}$-edge XANES, the strong white line originates from a dipole-allowed transition from core level $2 p$ to $5 d$, and the spectral profile is related to the unoccupied $\mathrm{Nd}$ $5 d$ states (Rao et al., 1983). As observed in Figure S4, the intensity of the white line at the $\mathrm{Nd} L_{3}$-edge XANES increases gradually with increasing uranium concentration. This can be mainly assigned to the strong covalent mixing of broad $\mathrm{U} 6 d$ with $\mathrm{O} 2 p$ competing with $\mathrm{Nd} 5 d-\mathrm{O} 2 p$ mixing. Furthermore, $\mathrm{U}$ doping leads to an increase in the symmetry of the $\mathrm{Nd}_{2} \mathrm{Zr}_{2} \mathrm{O}_{7}$ system and, in turn, a narrower $5 d$ band. Fig. 3(c) shows the $\mathrm{Zr} K$-edge XANES spectra of the NZO and U-doped NZO samples, which can be characterized by an obvious pre-edge structure (labelled by $a$ ) and a main peak split into two well resolved features (labelled by $b$ and $c$ ). Peaks $a$ and $b / c$ correspond to the electronic transition from the $1 s$ to $4 d$ orbital and the dipole-allowed transition from the $1 s$ to $5 p$ orbital, respectively (Komyoji et al., 1992; Popov et al., 2019). These features of all samples are close to those in the pyrochlore phase rather than in the defect-fluorite phase (Nandi et al., 2020). In Zr $K$-edge XANES, the pre-edge 
feature a for all samples is significant, which indicates tetrahedral distortion of the $\mathrm{ZrO}_{6}$ coordination polyhedron (Dargaud, Calas et al., 2010). The $c / b$ intensity ratios of all samples are similar to that of pyrochlore-type $\mathrm{Gd}_{2} \mathrm{Zr}_{2} \mathrm{O}_{7}$ with six-fold coordinated $\mathrm{Zr}^{4+}$. The ratio would rather deviate for $\mathrm{Zr}^{4+\delta}$ (NZO) compounds with seven- and eight-fold coordination (Komyoji et al., 1992; Galoisy et al., 1999; Li et al., 1993). Deeper knowledge of the local structure around $\mathrm{Zr}$ can be obtained from the quantitative analysis of EXAFS spectra. As shown in Fig. 3(d), all samples show two main coordination peaks, in which the first peak corresponds to six $\mathrm{O}_{48 f}$ atoms at approximately $2.2 \AA$, and the second peak corresponds to six $\mathrm{Zr}$ ions and six $\mathrm{Nd}$ ions. The quantitative results are shown in Table S4.

The $\mathrm{Zr} K$-edge EXAFS spectra are sensitive to changes in the local environment with increasing uranium content in the $\mathrm{Nd}_{2-x} \mathrm{U}_{x} \mathrm{Zr}_{2} \mathrm{O}_{7+\delta}$ and $\mathrm{Nd}_{2} \mathrm{Zr}_{2-y} \mathrm{U}_{y} \mathrm{O}_{7+\delta}$ samples. A noticeable change in the coordination number $(\mathrm{CN})$ is observed for $\mathrm{Zr}$ $\mathrm{O}_{48 f}$ bonds during uranium doping. Compared with that in NZO, the $\mathrm{CN}$ values of $\mathrm{Zr}-\mathrm{O}$ increased slightly in the $A-0.1$ and $B-0.1$ samples, which may be caused by the introduction of anions to maintain charge balance and preferentially fill the unoccupied $8 a$ site in the pyrochlore structure (Hess et al., 2002; Nästren et al., 2009). Since $\mathrm{Zr}$ is located at the $16 c$ site (adjacent to the $8 a$ site), the addition of $\mathrm{Zr}-\mathrm{O} \mathrm{CN}$ has little effect on the polyhedral structure of $\mathrm{Zr}-\mathrm{O}_{48 f}$. However, the $\mathrm{CN}$ of $\mathrm{Zr}-\mathrm{O}$ decreased significantly when keeping increasing the $\mathrm{U}$ content in $\mathrm{Nd}_{2-x} \mathrm{U}_{x} \mathrm{Zr}_{2} \mathrm{O}_{7+\delta}$ and $\mathrm{Nd}_{2} \mathrm{Zr}_{2-y} \mathrm{U}_{y} \mathrm{O}_{7+\delta}$. In this case, more uranium is introduced into pyrochlore, which causes the $\mathrm{Zr}-\mathrm{O}$ octahedron to be distorted, and the structure has more oxygen disorder characteristics by the movement of $\mathrm{O}_{48 f}$ and the formation of anionic Frenkel defects (Popov et al., 2019). In particular, this disorder is maximized at the highest doping concentration, which is consistent with the extremely low characteristic peak of pyrochlore in the Raman results. Through conventional $\mathrm{Zr} K$-edge EXAFS analysis, the pyrochlore and defect fluorite phases cannot be accurately distinguished. For the coordination of $\mathrm{Zr}-\mathrm{Zr}$ and $\mathrm{Zr}-\mathrm{Nd}, \mathrm{CN}$ decreases continuously with increasing uranium amount in the $\mathrm{Nd}_{2-x} \mathrm{U}_{x} \mathrm{Zr}_{2} \mathrm{O}_{7+\delta}$ and $\mathrm{Nd}_{2} \mathrm{Zr}_{2-y} \mathrm{U}_{y} \mathrm{O}_{7+\delta}$ systems, and the bond lengths of $\mathrm{Zr}-\mathrm{Zr}$ and $\mathrm{Zr}-\mathrm{La}$ increase regularly. This indicates that the movement of pyrochlore cation sites and the deformation of the pyrochlore lattice lead to the formation of a disordered system.

\section{Conclusion}

In this work, we systematically studied the uranium solubility, crystalline phase, valence state and structural evolution in a $\mathrm{Nd}_{2} \mathrm{Zr}_{2} \mathrm{O}_{7}$ pyrochlore synthesized by a modified molten salt method. According to the XRD results, uranium ions can be precisely confined at either the $\mathrm{Nd}$ or $\mathrm{Zr}$ sites in $\mathrm{Nd}_{2} \mathrm{Zr}_{2} \mathrm{O}_{7}$, and the solubility can reach up to a $20 \mathrm{~mol} \%$ substitution at the $\mathrm{Zr}$ sites and a $10 \mathrm{~mol} \%$ substitution at the $\mathrm{Nd}$ sites without any impurity phase. The Raman analysis shows that there is a phase transition from ordered pyrochlore to defective fluorite with increasing $U$ concentration. The TEM images show that all U-doped $\mathrm{Nd}_{2} \mathrm{Zr}_{2} \mathrm{O}_{7}$ samples have good crystallinity and dispersity except for $\mathrm{Nd}_{1.6} \mathrm{U}_{0.4} \mathrm{Zr}_{2} \mathrm{O}_{7+\delta}$. The $\mathrm{U} L_{3}$-edge XANES spectra indicate that uranium exists in the form of high-valent $\mathrm{U}^{6+}$ in all samples. To balance the extra charge from substituting $\mathrm{Nd}^{3+}$ or $\mathrm{Zr}^{4+}$ by high-valent $\mathrm{U}^{6+}$, additional oxygen is introduced along with a large structural distortion, as revealed by the XAFS analysis. This systematic work sheds light on understanding actinide immobilization, which will accelerate the development of advanced technology in nuclear waste storage materials.

\section{Acknowledgements}

We acknowledge the support from the beamline 14W1 of the Shanghai Synchrotron Radiation Facility (SSRF) and beamline 16A1 of National Synchrotron Radiation Research Center (NSRRC).

\section{Funding information}

The following funding is acknowledged: 'Transformational Technologies for Clean Energy and Demonstration', Strategic Priority Research Program of the Chinese Academy of Sciences (grant No. XDA2100000); the K. C. Wong Education Foundation (grant No. GJTD-2018-10); Youth Innovation Promotion Association, Chinese Academy of Science (grant No. Y201842); the National Science Foundation of China (grant No. 21876183); the National Science Foundation of China (grant No. 21771133); Instrument and Equipment Development Program Chinese Academy of Science (grant No. YJKYYQ20180066).

\section{References}

Abdou, M., Gupta, S. K., Zuniga, J. P. \& Mao, Y. (2018). Mater. Chem. Front. 2, 2201-2211.

Abdou, M., Gupta, S. K., Zuniga, J. P. \& Mao, Y. (2019). J. Lumin. 210, 425-434.

Blackburn, L. R., Sun, S.-K., Gardner, L. J., Maddrell, E. R., Stennett, M. C., Corkhill, C. L. \& Hyatt, N. C. (2021). J. Am. Ceram. Soc. 104, 2937-2951.

Conradson, S. D., Begg, B. D., Clark, D. L., den Auwer, C., Ding, M., Dorhout, P. K., Espinosa-Faller, F. J., Gordon, P. L., Haire, R. G., Hess, N. J., Hess, R. F., Keogh, D. W., Morales, L. A., Neu, M. P., Paviet-Hartmann, P., Runde, W., Tait, C. D., Veirs, D. K. \& Villella, P. M. (2004). J. Am. Chem. Soc. 126, 13443-13458.

Conradson, S. D., Begg, B. D., Clark, D. L., Den Auwer, C., EspinosaFaller, F. J., Gordon, P. L., Hess, N. J., Hess, R., Keogh, D. W., Morales, L. A., Neu, M. P., Runde, W., Tait, C. D., Veirs, D. K. \& Villella, P. M. (2003). Inorg. Chem. 42, 3715-3717.

Dargaud, O., Calas, G., Cormier, L., Galoisy, L., Jousseaume, C., Querel, G. \& Newville, M. (2010). J. Am. Ceram. Soc. 93, 342-344. Dargaud, O., Cormier, L., Menguy, N., Galoisy, L., Calas, G., Papin, S., Querel, G. \& Olivi, L. (2010). J. Non-Cryst. Solids, 356, 2928-2934. Ewing, R. C. (2011). Miner. Mag. 75, 2359-2377.

Finkeldei, S., Stennett, M. C., Kowalski, P. M., Ji, Y., de Visser-Týnová, E., Hyatt, N. C., Bosbach, D. \& Brandt, F. (2020). J. Mater. Chem. $A, \mathbf{8}, 2387-2403$.

Fuentes, A. F., Montemayor, S. M., Maczka, M., Lang, M., Ewing, R. C. \& Amador, U. (2018). Inorg. Chem. 57, 12093-12105.

Galoisy, L., Pélegrin, E., Arrio, M. A., Ildefonse, P., Calas, G., Ghaleb, D., Fillet, C. \& Pacaud, F. (1999). J. Am. Ceram. Soc. 82, 2219-2224. 
Garg, N., Pandey, K. K., Murli, C., Shanavas, K. V., Mandal, B. P., Tyagi, A. K. \& Sharma, S. M. (2008). Phys. Rev. B, 77, 214105.

Gregg, D. J., Zhang, Y., Zhang, Z., Karatchevtseva, I., Blackford, M. G., Triani, G., Lumpkin, G. R. \& Vance, E. R. (2013). J. Nucl. Mater. 438, 144-153.

Gupta, S. K. \& Mao, Y. (2021). Prog. Mater. Sci. 117, 100734.

Gupta, S. K., Reghukumar, C., Keskar, M. \& Kadam, R. M. (2016). J. Lumin. 177, 166-171.

Hagiwara, T., Nomura, K. \& Kageyama, H. (2019). Solid State Ion. 335, 32-37.

Hess, N. J., Begg, B. D., Conradson, S. D., McCready, D. E., Gassman, P. L. \& Weber, W. J. (2002). J. Phys. Chem. B, 106, 4663-4677.

Hu, Z. W., Kaindl, G. \& Meyer, G. (1997). J. Alloys Compd. 246, 186192.

Hu, Z. W., Kaindl, G. \& Meyer, G. (1998). J. Alloys Compd. 274, 38 41.

Jafar, M., Phapale, S. B., Mandal, B. P., Mishra, R. \& Tyagi, A. K. (2015). Inorg. Chem. 54, 9447-9457.

Komyoji, D., Yoshiasa, A., Moriga, T., Emura, S., Kanamaru, F. \& Koto, K. (1992). Solid State Ion. 50, 291-301.

Li, P., Chen, I. W. \& Penner-Hahn, J. E. (1993). Phys. Rev. B, 48, 10063-10073.

Lissner, F., Kramer, K., Schleid, T., Meyer, G., Hu, Z. W. \& Kaindl, G. (1994). Z. Anorg. Allg. Chem. 620, 444-450.

Liu, X., Fechler, N. \& Antonietti, M. (2013). Chem. Soc. Rev. 42, 8237-8265.

Liu, X., Pang, H., Liu, X., Li, Q., Zhang, N., Mao, L., Qiu, M., Hu, B., Yang, H. \& Wang, X. (2021). Innovation, 2, 100076.

Lu, X., Hou, C., Xie, Y., Shu, X., Ding, Y., Ma, D., Ren, W. \& Bian, L. (2017). Ceram. Int. 43, 3015-3024.

Lu, X., Shu, X., Chen, S., Zhang, K., Chi, F., Zhang, H., Shao, D. \& Mao, X. (2018). J. Hazard. Mater. 357, 424-430.

Lu, X., Shu, X., Shao, D., Chen, S., Zhang, H., Yuan, X. \& Chi, F. (2018). Ceram. Int. 44, 760-765.

Lu, X., Shu, X., Wang, L., Shao, D., Zhang, H., Zhang, K. \& Xie, Y. (2019). J. Alloys Compd. 771, 973-979.

Mączka, M., Sanjuán, M. L., Fuentes, A. F., Macalik, L., Hanuza, J., Matsuhira, K. \& Hiroi, Z. (2009). Phys. Rev. B, 79, 214437.

Manara, D. \& Renker, B. (2003). J. Nucl. Mater. 321, 233-237.

Mao, Y., Guo, X., Huang, J. Y., Wang, K. L. \& Chang, J. P. (2009). J. Phys. Chem. C, 113, 1204-1208.

Marlton, F. P., Zhang, Z., Zhang, Y., Proffen, T. E., Ling, C. D. \& Kennedy, B. J. (2021). Chem. Mater. 33, 1407-1415.

Martin, P. M., Belin, R. C., Valenza, P. J. \& Scheinost, A. C. (2009). J. Nucl. Mater. 385, 126-130.

Nandi, C., Poswal, A. K., Jafar, M., Kesari, S., Grover, V., Rao, R., Prakash, A. \& Behere, P. G. (2020). J. Nucl. Mater. 539, 152342.

Nästren, C., Jardin, R., Somers, J., Walter, M. \& Brendebach, B. (2009). J. Solid State Chem. 182, 1-7.
Niu, J., Wu, X., Zhang, H. \& Qin, S. (2019). RSC Adv. 9, 18954-18962. Palacios, M. L. \& Taylor, S. H. (2000). Appl. Spectrosc. 54, 1372-1378.

Park, S., Tracy, C. L., Zhang, F., Park, C., Trautmann, C., Tkachev, S. N., Lang, M., Mao, W. L. \& Ewing, R. C. (2018). Phys. Chem. Chem. Phys. 20, 6187-6197.

Pokhrel, M., Wahid, K. \& Mao, Y. (2016). J. Phys. Chem. C, 120, 14828-14839.

Popov, V. V., Menushenkov, A. P., Ivanov, A. A., Gaynanov, B. R., Yastrebtsev, A. A., d'Acapito, F., Puri, A., Castro, G. R., Shchetinin, I. V., Zheleznyi, M. V., Zubavichus, Y. V. \& Ponkratov, K. V. (2019). J. Phys. Chem. Solids, 130, 144-153.

Rao, K. J., Wong, J. \& Weber, M. J. (1983). J. Chem. Phys. 78, 62286237.

Ravel, B. \& Newville, M. (2005). J. Synchrotron Rad. 12, 537-541.

Sanjuán, M. L., Guglieri, C., Díaz-Moreno, S., Aquilanti, G., Fuentes, A. F., Olivi, L. \& Chaboy, J. (2011). Phys. Rev. B, 84, 104207.

Shu, X., Fan, L., Xie, Y., Zhu, W., Pan, S., Ding, Y., Chi, F., Wu, Y. \& Lu, X. (2017). J. Eur. Ceram. Soc. 37, 779-785.

Shu, X., Lu, X., Fan, L., Yang, R., Ding, Y., Pan, S., Zhou, P. \& Wu, Y. (2016). J. Mater. Sci. 51, 5281-5289.

Sun, J., Guo, X., Zhou, J., Dai, J., Song, S., Bao, H., Lin, J., Yu, H., He, S., Jiang, F., Long, D., Zhang, L. \& Wang, J.-Q. (2019). J. Synchrotron Rad. 26, 1733-1741.

Talanov, M. V. \& Talanov, V. M. (2021). Chem. Mater. 33, 2706-2725.

Tang, Z., Huang, Z., Han, W., Qi, J., Shi, Y., Ma, N., Zhang, Y., Guo, X. \& Lu, T. (2020). Inorg. Chem. 59, 9919-9926.

Toby, B. H. \& Von Dreele, R. B. (2013). J. Appl. Cryst. 46, 544-549.

Turner, K. M., Rittman, D. R., Heymach, R. A., Tracy, C. L., Turner, M. L., Fuentes, A. F., Mao, W. L. \& Ewing, R. C. (2017). J. Phys. Condens. Matter, 29, 255401.

Wang, L., Li, J., Xie, H., Chen, Q. \& Xie, Y. (2021). Prog. Nucl. Energy, 137, 103774.

Wang, L.-L., Li, J.-B., Xie, H., Deng, S.-H., Zhang, K.-X. \& Yi, F.-C. (2018). Acta Phys. Sin. 67, 192801.

Wang, Y., Darapaneni, P., Ofoegbuna, T., Gupta, S. K., Kizilkaya, O., Mao, Y. \& Dorman, J. A. (2020). Inorg. Chem. 59, 14070-14077.

Zhang, F. X., Lang, M., Liu, Z. \& Ewing, R. C. (2010). Phys. Rev. Lett. 105, 015503.

Zhang, F. X., Lang, M., Tracy, C., Ewing, R. C., Gregg, D. J. \& Lumpkin, G. R. (2014). J. Solid State Chem. 219, 49-54.

Zhang, L., Qie, M., Su, J., Zhang, S., Zhou, J., Li, J., Wang, Y., Yang, S., Wang, S., Li, J., Wu, G. \& Wang, J.-Q. (2018). J. Synchrotron Rad. 25, 514-522.

Zhang, L., Su, J., Yang, S., Guo, X., Jia, Y., Chen, N., Zhou, J., Zhang, S., Wang, S., Li, J., Li, J., Wu, G. \& Wang, J.-Q. (2016). Ind. Eng. Chem. Res. 55, 4224-4230.

Zhang, L., Zhou, J., Zhang, J., Su, J., Zhang, S., Chen, N., Jia, Y., Li, J., Wang, Y. \& Wang, J.-Q. (2016). J. Synchrotron Rad. 23, 758-768.

Ziolkowski, J. (1985). J. Solid State Chem. 57, 269-290. 